\title{
Efeito do Acondicionamento Térmico Ambiental sobre o Desempenho Reprodutivo da Fêmea Suína
}

\author{
Christiane Garcia Vilela Nunes ${ }^{1}$, Eduardo Paulino da Costa ${ }^{2}$, Rita Flávia Miranda de Oliveira ${ }^{3}$, \\ Juarez Lopes Donzele ${ }^{3}$, Ricardo Vianna Nunes ${ }^{1}$, Giovanni Ribeiro de Carvalho ${ }^{3}$
}

RESUMO - O objetivo deste experimento foi avaliar um sistema de acondicionamento térmico artificial para salas de porcas em gestação, em Ponte Nova-MG. Foram utilizadas 95 porcas Cambourough 22® (matriz comercial da Agroceres $\AA$ ), de segundo parto em diante, distribuídas em dois tratamentos. No tratamento 1, foram utilizadas 46 matrizes, submetidas, nos primeiros 35 dias de gestação, a um sistema de acondicionamento térmico artificial com o uso de ventiladores e nebulizadores, acionados automaticamente de acordo com a temperatura do ar (VFN). No tratamento 2, foram utilizadas 49 porcas submetidas, nos primeiros 35 dias de gestação, a um ambiente sem qualquer sistema de acondicionamento térmico artificial (SVFN). Após os 35 dias de gestação, todos os animais receberam o mesmo manejo até o parto. A temperatura média ambiente, para os tratamentos VFN e SVFN, foi de 22,6 e 23, $4^{\circ} \mathrm{C}$, respectivamente. Nas horas mais quentes do dia, o sistema de acondicionamento térmico foi eficiente em reduzir a temperatura do ar em $2^{\circ} \mathrm{C}$. Apesar dessa diferença, o VFN não foi eficiente em reduzir a temperatura ambiente para a temperatura de conforto dos animais $\left(18-20^{\circ} \mathrm{C}\right)$; em ambos os tratamentos, os animais apresentaram frequência respiratória elevada. Entretanto, esta foi eficiente na manutenção da temperatura retal, o que significa que as porcas não foram submetidas a uma condição muito severa de estresse por calor, que justificasse a utilização do sistema de acondicionamento empregado. Ainda, para os parâmetros de eficiência reprodutiva, não foi observada diferença entre os tratamentos. Os níveis de progesterona no $6^{0}$ e $13^{\circ}$ dia pós-cobertura estavam dentro da normalidade para a espécie suína. Assim, para a época do outono, na região de Ponte Nova-MG, o sistema de acondicionamento térmico constituído por ventilação e nebulização não incrementou a eficiência reprodutiva.

Palavras-chave: acondicionamento térmico, eficiência reprodutiva, suínos

\section{Effect of a System with Thermic Acondition Environment on Reproductive Efficiency of Female Swine}

\begin{abstract}
The objetive of this experiment was to evaluate a system of thermal artificial condition for gestation sow housing, at Ponte Nova-MG. A total of 95 Cambourough $22 \AA$ sows from the second parturition were assigned to two treatments. Forty-six sows of treatment one were submitted, during the first 35 days of gestation, to a system of controlled thermal artificial condition (fans and aspersion) which were automatically turned on according to the air temperature (VFN). In treatment two, during the same period 49 sows of the same variety as the first ones were submitted to any artificial thermal condition (SVFN). From 35 days on of gestation to the parturition, all the animals received the same management. The average room temperature for treatments VFN and SVFN were $22.6^{\circ} \mathrm{C}$ and $23.4^{\circ} \mathrm{C}$, respectively. During the hottest hours of the day, this system was efficient to reduce the temperature by $2^{\circ} \mathrm{C}$. In spite of such difference, the system could not reduce the room temperature to an adequate level to these animals $\left(18-21^{\circ} \mathrm{C}\right)$. For this reason the animals showed high breathing frequency in both treatments, but this was capable to maintain the body temperature of the sows. There were no differences on the evaluated parameters of reprodutive efficiency and progesterone levels. That is why the ventilation and nebulization systems during the autumn is not justified since the temperatures at this time of the year is not high enough to take the animals to a stress condition which could directly affect their reproductive performance.
\end{abstract}

Key Words: reproductive efficiency, thermic acondition, swine

\section{Introdução}

As condições ambientais inadequadas, que são uma resposta direta do clima externo, afetam consideravelmente a produção e a reprodução nos suínos. De acordo com Lee \& Phillips (1948), os suínos são, entre os animais domésticos, os mais sensíveis a altas temperaturas, o que se deve ao seu metabolismo elevado, à camada de tecido adiposo, além do sistema temorregulador pouco desenvolvido. Ainda segundo estes pesquisadores, os suínos morrem de hipertermia, quando sua temperatura retal atinge $44,5^{\circ} \mathrm{C}$, uma vez que não suam.

\footnotetext{
${ }^{1}$ Alunos de Doutorado - UFV.

${ }^{2}$ Professor Adjunto - UFV - DVT - Bolsista de produtividade de pesquisa - CNPq. E.mail: epcosta@ufv.br

3 Professores Adjunto - UFV - DZO.
} 
Segundo Moura (1999), a faixa de temperatura ideal para porcas em gestação se encontra entre 18 e $21^{\circ} \mathrm{C}$ e a umidade relativa, entre 50 e $70 \%$. Temperaturas acima dessa faixa têm sido associadas a falhas reprodutivas e perdas econômicas na produção. Assim, Love (1978, 1981), Stork (1979), Hurtgen \& Leman (1980) e Rozeboom et al. (2000) documentaram a ocorrência, em épocas quentes e úmidas do ano, da "Síndrome da Infertilidade de Verão". Esta condição é caracterizada por anestro, aumento do intervalo desmama-cobertura, retorno ao estro, baixa taxa de parição e concepção e alta taxa de abortamento.

$\mathrm{O}$ estresse causado por temperaturas ambientes elevadas parece ser um dos principais fatores responsáveis pela baixa eficiência reprodutiva nessas épocas do ano. Segundo Wetteman \& Bazer (1985), Varley (1993) e Van Der Lende (2000), temperaturas ambientes elevadas levam à mortalidade embrionária no primeiro mês de gestação e, conseqüentemente, à menor taxa de parto (Love, 1978, 1981) e menor tamanho da leitegada (Domínguez et al., 1996).

Como o suíno adulto é mais resistente ao frio do que ao calor, em regiões de clima mais quente, há necessidade de climatização (Nääs, 2000). Este autor recomenda a utilização de ventiladores nas instalações, uma vez que a temperatura crítica de tolerância ao calor é beneficiada pelo acionamento do mecanismo de troca térmica de convecção, devido à ventilação. Porém, muitas vezes, somente a presença de ventiladores não é suficiente para se alcançar as condições ideais mínimas de conforto, havendo a necessidade também do uso de nebulização.

Dessa forma, este trabalho foi conduzido objetivando-se a avaliação de um sistema de acondicionamento térmico, utilizando ventilação forçada associada à nebulização em salas de gestação de suínos, e de seu efeito sobre a eficiência reprodutiva da fêmea.

\section{Material e Métodos}

O experimento foi realizado em uma granja suinícola localizada na região de Ponte Nova-MG, no período de março a agosto de 2000 . A cidade está localizada a uma latitude de $20^{\circ} 23^{\prime}$ sul e longitude $43^{\circ}$ $01^{\prime}$ oeste, com altitude de $540 \mathrm{~m}$. O clima da região, de acordo com a classificação de Köppen, é Cwa (quente, temperado, chuvoso, com estação seca no inverno e verão quente).

Foram utilizadas 95 porcas Camborough $22 \AA$ a partir do segundo parto, distribuídas nos tratamentos de acordo com a ordem de parto: porcas de segundo parto; porcas de terceiro a quinto parto; e porcas com mais de seis partos. Todas as porcas entraram nos tratamentos com a mesma condição corporal.

A medição da espessura de toucinho foi realizada com um aparelho de ultra-som, posicionado na altura da $10^{a}$ costela, a $6 \mathrm{~cm}$ da linha média. Foi medida a espessura de toucinho de duas e três camadas de gordura. O escore corporal utilizado foi de 1 a 5 , sendo: 1 - muito magra; 2 - magra; 3 - bom; 4 - gorda; 5 - obesa (Agroceres, 2000). A avaliação foi realizada por uma única pessoa, em todos os animais e tratamentos.

O experimento foi conduzido no galpão de gestação da granja, construído de alvenaria, nas seguintes dimensões: $126 \mathrm{~m}$ de comprimento, 5,8 $\mathrm{m}$ de largura e 3,0 m de altura, com cobertura de telha de amianto provida de lanternim. As laterais eram abertas e providas de uma mureta de $0,8 \mathrm{~m}$ de altura.

O galpão foi dividido no sentido transversal, com

Tabela 1 - Valores médios de condição corporal e de espessura de toucinho na segunda e terceira camadas de gordura das porcas durante a cobertura, para os respectivos tratamentos (VFN e SVFN)

Table 1 - Average values for score condition and backfat level on the second and third fat layers of sows for the respective treatments (VFN and SVFN)

\begin{tabular}{|c|c|c|c|}
\hline \multirow[t]{2}{*}{$\begin{array}{l}\text { Parâmetros } \\
\text { Parameters }\end{array}$} & \multicolumn{2}{|c|}{$\begin{array}{l}\text { Tratamentos } \\
\text { Treatments }\end{array}$} & \multirow[t]{2}{*}{$\begin{array}{l}\text { Média } \\
\text { Average }\end{array}$} \\
\hline & VFN & SVFN & \\
\hline $\begin{array}{l}\text { Escore corporal } \\
\text { Score condition }\end{array}$ & $2,8 \pm 0,4$ & $2,8 \pm 0,4$ & $2,8 \pm 0,4$ \\
\hline $\begin{array}{l}\text { Espessura de toucinho na } 2 \text { a camada }(\mathrm{mm}) \\
\text { Back fat level on the second layer }(\mathrm{mm})\end{array}$ & $14 \pm 3,9$ & $13 \pm 3,9$ & $13,5 \pm 3,9$ \\
\hline $\begin{array}{l}\text { Espessura de toucinho na } 3 \text { a camada }(\mathrm{mm}) \\
\text { Backfat level on the third layer }(\mathrm{mm})\end{array}$ & $21 \pm 5,8$ & $18 \pm 5,4$ & $19,5 \pm 5,7$ \\
\hline
\end{tabular}

R. Bras. Zootec., v.32, n.4, p.854-863, 2003 
lona de PVC, em dois ambientes, de maneira a não permitir trocas de ar entre as duas unidades ambientais. Em um dos ambientes foi montado o sistema de ventilação forçada, associada à nebulização. Foram utilizados oito ventiladores do tipo axial de 30 polegadas, dispostos em uma das laterais do galpão, posicionados a favor da direção do vento. Os ventiladores foram colocados à altura de $1,60 \mathrm{~m}$ do solo e distanciados 3,25 $\mathrm{m}$ um do outro. A quantidade de ventiladores foi calculada de acordo com as dimensões do galpão, a capacidade dos ventiladores e a categoria animal. Foram utilizadas duas linhas de nebulização, posicionadas da seguinte forma: altura do solo de 2,0 m, estando a primeira linha $50 \mathrm{~cm}$ à frente da linha dos ventiladores, e a segunda no corredor, entre a primeira e a segunda fileira de porcas. Oito bicos de nebulização foram utilizados em cada linha, um na frente de cada ventilador. O sistema de ventilação forçada associada à nebulização foi acionado automaticamente, da seguinte forma: os ventiladores eram ligados quando a temperatura ultrapassava $21^{\circ} \mathrm{C}$ e a nebulização, quando a temperatura ultrapassava $24^{\circ} \mathrm{C}$. A nebulização era desligada quando a umidade relativa do ar ultrapassava $70 \%$. O controle do sistema era realizado por meio de um termostato e um umidostato, posicionados no centro do galpão, à meia altura do corpo dos animais. No outro ambiente, não foi utilizado nenhum sistema de acondicionamento térmico artificial.

Para o tratamento 1 foram utilizadas 46 porcas, alojadas em gaiolas individuais de gestação, em um ambiente com um sistema de ventilação forçada e de nebulização (VFN), controlados automaticamente de acordo com a temperatura do ambiente e a umidade relativa do ar. No tratamento 2 foram utilizadas 49 porcas, distribuídas de acordo com a ordem de parto. Estas porcas foram alojadas em gaiolas individuais de gestação, em um ambiente sem ventilação forçada e nebulização (SVFN).

As porcas foram alojadas no galpão de gestação no momento da primeira inseminação. Foram realizadas três inseminações por porca, sendo a primeira 12 horas após o início do estro (aceitação do macho pela fêmea); a segunda, 12 horas após a primeira; e a terceira, 12 horas após a segunda. As porcas que continuaram apresentando sinais de estro foram inseminadas uma quarta vez. Somente foi utilizado sêmen que apresentasse motilidade e vigor espermáticos acima de $80 \%$ e 4 , respectivamente. Após um período de 35 dias, todas as porcas de ambos os tratamentos foram transferidas para outro galpão de gestação, sem qualquer sistema de acondicionamento térmico artificial, onde permaneceram até o parto.

A alimentação das porcas da cobertura até o 35으 dia de gestação foi constituída por uma ração de gestação contendo $2.986 \mathrm{kcal} / \mathrm{kg}$ de ED, a qual foi fornecida na quantidade de $2,2 \mathrm{~kg} / \mathrm{dia}$, duas vezes ao dia, às $6 h 30$ e 15 h30. Do 360 dia de gestação até o parto, os animais de todos os tratamentos receberam o mesmo manejo nutricional.

Em cada galpão foram instalados um termômetro de globo negro e um psicrômetro, a $0,40 \mathrm{~m}$ do piso, correspondendo à altura média da porca.

Os dados de temperatura do globo negro e os da temperatura de bulbo seco (TBS) e bulbo úmido, obtidos por meio do psicrômetro, foram utilizados para determinação da umidade relativa do ar (UR) e do índice de temperatura de globo e umidade (ITGU), segundo Buffington et al. (1981). Para obtenção de dados representativos do ambiente externo, foi instalado na área externa um abrigo meteorológico contendo um psicrômetro no seu interior e, externamente, um termômetro de globo negro. Os dados obtidos foram posteriormente utilizados para determinação do ITGU.

A coleta dos dados referentes ao meio ambiente foi realizada durante os primeiros 35 dias de gestação. Os dados dos ambientes térmicos, interno e externo, foram coletados nos horários de $6,10,14 \mathrm{e}$ $18 \mathrm{~h}$, a cada dois dias, e nos horários de 2, 6, 10, 14, 18 e $22 \mathrm{~h}$, a cada sete dias.

Com base nos dados coletados em cada horário, para cada tratamento e para o ambiente externo, foram calculados a umidade relativa do ar (UR) e o índice de temperatura de globo e umidade (ITGU). Para obtenção dos valores de UR e ITGU, foram utilizadas as seguintes fórmulas:

1) $\mathrm{UR}=\mathrm{f} \cong \mathrm{e} / \mathrm{e}_{\mathrm{s}} \times 100$ (Vianello \& Alves, 1991) em que: $\mathrm{e}=$ pressão real de vapor; e $\mathrm{e}_{\mathrm{s}}=$ pressão de saturação.

2) $\mathrm{ITGU}=\mathrm{Tgn}+0,36 \mathrm{Tpo}-330,08$ (Buffington et al., 1981)

em que: $\operatorname{Tgn}=$ temperatura de globo negro, $\mathrm{K}$; e Tpo = temperatura do ponto de orvalho, $\mathrm{K}$.

A temperatura retal (TR) e a freqüência respiratória (FR) das porcas foram tomadas a cada sete dias, às $9 \mathrm{~h}$, para caracterizar as respostas fisiológicas no período da manhã, e às $15 \mathrm{~h}$, para caracterizar as respostas no período da tarde. Para medição da temperatura retal, foi utilizado um termômetro digital

\section{R. Bras. Zootec., v.32, n.4, p.854-863, 2003}


da marca comercial BD, introduzido no reto do animal, e para a freqüência respiratória foram contados os movimentos do flanco das porcas por 30 segundos e realizada a correção para um minuto.

Foi coletado sangue dos animais, no 6o e 130 dia de gestação, por meio de punção no sínus orbital (Friend \& Brown, 1971). O sangue coletado, após permanecer em repouso por 40 minutos, foi centrifugado a $1.500 \mathrm{~g}$, por um período de 10 minutos, para retirada do soro, o qual foi armazenado em congelador. Após esse processo, foi realizada a determinação dos níveis séricos do hormônio progesterona, por meio de "kit" de determinação, utilizando a técnica de radioimunoensaio.

Ao término do período experimental, foram coletados dados de repetição de estro, número de partos, tamanho da leitegada, número de leitões nascidos vivos, natimortos e mumificados. Os dados de repetição de estro e número de parições foram utilizados para o cálculo da taxa de repetição de estro, da taxa de repetição de estro regular e irregular e da taxa de parição. Para o cálculo da taxa de repetição de estro regular, foram consideradas as repetições entre 18 e 24 e 39 e 45 dias pós-cobertura e para a taxa de repetição irregular, as repetições entre 25 e 38 e acima de 45 dias pós-cobertura

Durante o período experimental, foram anotados os abortos visualmente detectados, para o cálculo da taxa de abortamento.

$\mathrm{O}$ experimento foi montado em um esquema de parcelas subdivididas, tendo nas parcelas os tratamentos (ambiente SVN e ambiente SVFN) e nas subparcelas os horários $(2,6,10,14,18,22$ h), em um delineamento em blocos casualizados com 35 repetições, sendo o bloco cada dia.

Os dados foram submetidos às análises de variância e regressão, utilizando o programa SAEG (1999). Para o fator qualitativo (tratamentos), as médias foram comparadas por meio do teste de Tukey, a 5\% de probabilidade. Para o fator quantitativo (dados relativos aos horários), os modelos foram escolhidos com base na significância dos coeficientes de regressão, utilizando o teste "t", a $1 \%$ de probabilidade, no coeficiente de determinação $\left(\mathrm{r}^{2}=\right.$ SQRegressão/SQHoras) e no fenômeno em estudo.

Para as variáveis freqüência respiratória (FR) e temperatura retal (TR), os dados foram analisados segundo o esquema de parcelas subdivididas, tendo nas parcelas os tratamentos (ambientes SVN e SVFN) e nas subparcelas os períodos (manhã e tarde), em um delineamento em blocos casualizados, com seis repetições, sendo o dia da coleta considerado um bloco. Os dados foram submetidos a ANOVA. As médias foram comparadas, utilizando o teste Tukey, a $5 \%$ de probabilidade.

Para comparação das variáveis qualitativas (taxa de repetição de estro, taxa de parição e de abortamento) entre os tratamentos, foi utilizado o teste de qui-quadrado. Para comparação das variáveis quantitativas (número de leitões nascidos totais por porca, número de leitões nascidos vivos, natimortos, mumificados e concentração de progesterona), os dados foram submetidos inicialmente ao teste de normalidade (teste de Lilliefors) e de homocedasticidade (teste de Cochran \& Bartlett). Posteriormente, as variáveis foram interpretadas pela análise de variância (ANOVAG) e submetidas ao teste de Tukey; as médias dos tratamentos foram comparadas a $5 \%$ de probabililidade.

\section{Resultados e Discussão}

Observou-se diferença $(\mathrm{P}<0,01)$ entre os tratamentos, para as horas e as interações Horas x Tratamento, para o TBS, UR e ITGU. Como neste experimento o interesse concentrou-se nos efeitos dos tratamentos, passou-se, então, a estudar a interação HS x TRAT para todas as variáveis. Esses dados podem ser observados na Tabela 2. Observa-se que, nos horários de 10 e $14 \mathrm{~h}$, houve diferença $(\mathrm{P}<0,05)$ entre os tratamentos VFN e SVFN, para as variáveis estudadas, exceto para a UR às $14 \mathrm{~h}$. As diferenças encontradas devem-se, provavelmente, ao sistema de ventilação forçada associada à nebulização, que nesses horários do dia já se encontravam acionados e funcionando de forma mais eficiente, devido aos menores valores da UR do ar.

Verificou-se que os valores de TBS foram menores nos horários de 2 e $6 \mathrm{~h}$, para todos os tratamentos. Nesses horários, não houve diferença entre os tratamentos para essa variável, o que já era esperado, uma vez que a temperatura se encontrava abaixo de $21^{\circ} \mathrm{C}$, não sendo acionados os ventiladores e nebulizadores. Nos horários de 10 e 14 h, os valores de TBS foram menores no tratamento VFN, quando comparados aos do tratamento SVFN. Entretanto, o sistema foi mais eficiente às $14 \mathrm{~h}$, quando o valor de TBS estava mais alto e a UR mais baixa. Provavelmente, a maior eficiência do sistema nesse horário deve-se aos menores valores de UR, ficando os nebulizadores

R. Bras. Zootec., v.32, n.4, p.854-863, 2003 
Tabela 2 - Valores médios observados de temperatura de bulbo seco (TBS), umidade relativa (UR) e índice de temperatura de globo e umidade (ITGU), para os respectivos tratamentos e horas

Table 2 - Avarage values for dry bulb temperature (DBT), relative humidity (RH) and black globe humidity index (BGHI), for the respective treatments and hours

\begin{tabular}{|c|c|c|c|c|c|c|}
\hline \multirow[t]{2}{*}{$\begin{array}{l}\text { Tratamento } \\
\text { Treatment }\end{array}$} & \multicolumn{6}{|c|}{$\begin{array}{l}\text { Hora } \\
\text { Hour }\end{array}$} \\
\hline & $2: 00$ & $6: 00$ & $10: 00$ & $14: 00$ & $18: 00$ & $22: 00$ \\
\hline & \multicolumn{5}{|c|}{ Temperatura de bulbo seco $\left({ }^{\circ} \mathrm{C}\right)$} & Dry bulb temperature \\
\hline $\mathrm{VFN}^{1}$ & $19,62^{\mathrm{A}}$ & $19,19^{\mathrm{A}}$ & $24,06^{\mathrm{B}}$ & $26,50^{\mathrm{C}}$ & $24,80^{\mathrm{A}}$ & $21,57^{\mathrm{B}}$ \\
\hline $\mathrm{SVFN}^{2}$ & $19,64^{\mathrm{A}}$ & $19,39^{\mathrm{A}}$ & $25,14^{\mathrm{A}}$ & $28,49^{\mathrm{B}}$ & $25,26^{\mathrm{A}}$ & $22,40^{\mathrm{A}}$ \\
\hline \multirow[t]{2}{*}{$\mathrm{EXT}^{3}$} & $19,01^{\mathrm{A}}$ & $17,32^{\mathrm{B}}$ & $25,38^{\mathrm{A}}$ & $29,50^{\mathrm{A}}$ & $25,35^{\mathrm{A}}$ & $20,60^{C}$ \\
\hline & \multicolumn{6}{|c|}{$\begin{array}{l}\text { Umidade relativa }(\%) \\
\text { Relative humidity }\end{array}$} \\
\hline VFN & $91,87^{\mathrm{AB}}$ & $90,70^{\mathrm{B}}$ & $76,56^{\mathrm{A}}$ & $65,75^{\mathrm{B}}$ & $71,16^{\mathrm{C}}$ & $92,15 \mathrm{~A}$ \\
\hline SVFN & $91,63^{\mathrm{B}}$ & $89,71^{\mathrm{B}}$ & $72,49^{\mathrm{B}}$ & $63,14^{\mathrm{B}}$ & $75,47^{\mathrm{B}}$ & $84,90 \mathrm{~B}$ \\
\hline \multirow[t]{2}{*}{ EXT } & $94,65^{\mathrm{A}}$ & $95,27^{\mathrm{A}}$ & $77,55^{\mathrm{A}}$ & $68,82^{\mathrm{A}}$ & $81,22^{\mathrm{A}}$ & $92,99 \mathrm{~A}$ \\
\hline & \multicolumn{6}{|c|}{$\begin{array}{l}\text { Índice de temperatura de globo e umidade } \\
\text { Black globe humidity index }\end{array}$} \\
\hline VFN & $67,62^{\mathrm{A}}$ & $66,76^{\mathrm{A}}$ & $72,40^{\mathrm{C}}$ & $74,39^{\mathrm{C}}$ & $72,57^{\mathrm{B}}$ & $69,80^{\mathrm{A}}$ \\
\hline SVFN & $68,02^{\mathrm{A}}$ & $67,66^{\mathrm{A}}$ & $73,76^{\mathrm{B}}$ & $76,98^{\mathrm{B}}$ & $73,78^{\mathrm{A}}$ & $70,50^{\mathrm{A}}$ \\
\hline EXT & $65,46^{\mathrm{B}}$ & $64,00^{\mathrm{B}}$ & $87,98^{\mathrm{A}}$ & $93,29^{\mathrm{A}}$ & $71,78^{\mathrm{B}}$ & $66,54^{\mathrm{B}}$ \\
\hline
\end{tabular}

Médias, na coluna, seguidas de letras diferentes, diferem $(P<0,05)$ pelo teste Tukey.

Means in the column followed by different letter are different by Tukey test).

1 VFN - ambiente com ventilação e nebulização (environment with ventilation and nebulization).

2 SVFN - ambiente sem ventilação e nebulização (environment with no ventilation and nebulization).

3 EXT - ambiente externo (outside enviroment).

ligados por mais tempo, o que, segundo Baêta \& Souza (1997), pode ter favorecido a dissipação de calor na forma latente, em razão da menor pressão de vapor de água (UR). Estes resultados estão de acordo com aqueles obtidos por Turco (1993), que, trabalhando com diferentes sistemas de acondicionamento térmico para porcas, também encontrou menores valores para TBS nos horários mais quentes do dia, quando foram observados menores valores de UR.

Às $10 \mathrm{~h}$, observou-se que o sistema de ventilação forçada associada à nebulização não foi tão eficiente em abaixar a temperatura do ar, o que pode ser explicado pelos altos valores de UR, o que limitou a utilização da nebulização, ficando somente os ventiladores ligados.

Segundo Nääs (2000), a função da nebulização é reduzir a temperatura no interior da instalação, sendo o limite de redução relacionado diretamente aos valores de UR. Em climas com altas UR, em torno de $80-90 \%$, a nebulização deve ser usada com maior freqüência nas horas mais quentes do dia, o que, geralmente, coincide com o período de umidades relativas mais baixas.

Comparando as médias de ITGU para cada tratamento, em cada horário, constatou-se que, nos horá- rios de maior radiação, das 10 às $14 \mathrm{~h}$, houve diferença $(\mathrm{P}<0,05)$ entre os tratamentos. A maior diferença foi observada no horário mais quente do dia $(14 \mathrm{~h})$. Os valores obtidos de ITGU evidenciaram a maior eficiência do sistema de ventilação forçada associada à nebulização nos horários mais quentes do dia, quando ambas ventilação e nebulização estavam acionadas. Entretanto, os valores de ITGU em ambos os ambientes, nos horários de 10, 14 e 18 h, encontravam-se, segundo trabalho realizado por Turco (1993), acima do ITGU crítico superior de matrizes suínas. Esse autor relatou que o ITGU crítico superior das porcas está em torno de 72. Acima deste valor, o autor verificou aumento sustentado da freqüência respiratória (FR) e da temperatura retal (TR), presumindo-se a ocorrência de desconforto térmico para as porcas. No entanto, neste estudo, os valores de ITGU, mesmo nos horários mais quentes do dia, não atingiram o crítico para as porcas, uma vez que, os animais não apresentaram a TR elevada (Tabela 4).

Com base nos resultados apresentados na Tabela 2, foram obtidas as equações de regressão (Tabela 3) referentes aos dados de TBS, UR e ITGU com relação aos tratamentos (TRAT) e ao ambiente 
externo (EXT), em função das horas observadas, sendo o modelo cúbico-raiz o que melhor se ajustou para todas as variáveis estudadas.

Observou-se que a média da temperatura do ar para todos os ambientes foi maior por volta de $15 \mathrm{~h}$. Entretanto, o ambiente com VFN apresentou, para esse horário, diferença de menos $2^{\circ} \mathrm{C}\left(27 \times 25^{\circ} \mathrm{C}\right)$ na temperatura do ar, quando comparado ao ambiente SVFN, e de menos $3,5^{\circ} \mathrm{C}\left(28,5 \times 25^{\circ} \mathrm{C}\right)$, quando comparado ao ambiente EXT. Estas diferenças se devem, provavelmente, à melhor eficiência do sistema de ventilação forçada associada à nebulização nos horários mais quentes do dia. Observou-se que, das $15 \mathrm{~h}$ em diante, a diferença na temperatura do ar, entre os tratamentos reduzia, o que pode ser explicado pela menor eficiência do sistema nesses horários, uma vez que a temperatura ambiente diminui e a UR aumenta, o que leva, conseqüentemente, à menor freqüência de acionamento dos nebulizadores. Nos horários entre 23 e $7 \mathrm{~h}$, quando a temperatura do ar não ultrapassou $21^{\circ} \mathrm{C}$, as temperaturas dos ambientes VFN e SVFN apresentaram-se semelhantes, o que pode ser justificado pelo não-acionamento dos ventiladores e nebulizadores nesses horários.

Não foi observado efeito da interação $(\mathrm{P}>0,05)$ para quaisquer dos parâmetros fisiológicos avalia- dos. Entretanto, observou-se que a variável FR foi influenciada $(\mathrm{P}<0,05)$ pelos tratamentos, enquanto a TR variou $(\mathrm{P}<0,05)$ somente entre os períodos.

$\mathrm{Na}$ Tabela 4, estão os dados de freqüência respiratória e temperatura retal dos animais, em razão do período (manhã e tarde) e dos tratamentos. Não foi observado efeito $(\mathrm{P}>0,05)$ do período (manhã e tarde) sobre a FR dos animais. No entanto, constatou-se que os animais mantidos no tratamento SVFN tiveram, em valor absoluto, a FR 32\% maior que aqueles no tratamento VFN, no período da tarde.

Essa diferença pode ter sido atribuída às maiores temperaturas do ar no ambiente SVFN. Entretanto, em ambos os ambientes, a temperatura foi suficiente para aumentar a FR dos animais a valores acima do considerado normal para porcas em gestação ( 15 a 20 por minuto). De acordo com os valores encontrados para a FR e a TR, pode-se observar que a FR estava acima do valor normal para a espécie suína, em ambos os tratamentos. Entretanto, o aumento da FR, em ambos os tratamentos, foi suficiente para a dissipação do calor e a manutenção da TR dos animais, sugerindo que a condição de estresse pelo calor não foi muito severa. Segundo Hannas (1999), a taxa respiratória para fêmeas gestantes, em condições ambientais de termoneutralidade, está em torno de 15

Tabela 3 - Equações de regressão ajustadas das variáveis temperatura de bulbo seco (TBS), umidade relativa (UR) e índice de temperatura de globo e umidade (ITGU) em função da hora $(\mathrm{H})$, para os respectivos tratamentos e coeficientes de determinação

Table 3 - Regression equations adjusted by dry-bulb temperature (DBT), relative humidity (RH) and black globe humidity index (BGHI) variables due to time $(H)$, for the respective treatments and determinated coefficients

\begin{tabular}{lcc}
\hline $\begin{array}{l}\text { Tratamentos } \\
\text { Treatments }\end{array}$ & $\begin{array}{c}\text { Equações ajustadas } \\
\text { Adjusted equations }\end{array}$ & $\mathrm{R}^{2}$ \\
\hline (TBS) VFN & $\hat{y}=54,570-46,246^{* *} \sqrt{H}+18,154 \mathrm{H}^{* *}-2,091^{* *} \sqrt{H^{3}}$ & 0,98 \\
(TBS) SVFN & $\hat{y}=58,332-51,668^{* *} \sqrt{H}+20,501 \mathrm{H}^{* *}-2,376^{* *} \sqrt{H^{3}}$ & 0,94 \\
(TBA) EXT & $\hat{y}=79,028-78,813^{* *} \sqrt{H}+30,630 \mathrm{H}^{* *}-3,521^{* *} \sqrt{H^{3}}$ & 0,95 \\
(UR)VFN & $\hat{y}=-51,820+190,734^{* *} \sqrt{H}-75,607 \mathrm{H}^{* *}+8,841^{* *} \sqrt{H^{3}}$ & 0,99 \\
(UR) SVFN & $\hat{y}=-12,487+142,688^{* *} \sqrt{H}-58,497 \mathrm{H}^{* *}+6,948^{* *} \sqrt{H^{3}}$ & 0,93 \\
(UR)EXT & $\hat{y}=-26,638+163,083^{* *} \sqrt{H}-65,411 \mathrm{H}^{* *}+7,710^{* *} \sqrt{H^{3}}$ & 0,94 \\
(ITGU) VFN & $\hat{y}=103,191-47,021^{* *} \sqrt{H}+18,395 \mathrm{H}^{* *}-2,112^{* *} \sqrt{H^{3}}$ & 0,96 \\
(ITGU)SVFN & $\hat{y}=108,945-54,605^{* *} \sqrt{H}+21,649 \mathrm{H}^{* *}-2,511^{* *} \sqrt{H^{3}}$ & 0,95 \\
(ITGU)EXT & $\hat{y}=170,612-146,047 * * \sqrt{H}+60,606 \mathrm{H}^{* *}-7,332 * * \sqrt{H^{3}}$ & 0,76
\end{tabular}

** Significativo $(\mathrm{P}<0,01)$, pelo teste "t"(** Significant by "t" test).

1 VFN - ambiente com ventilação e nebulização (environment with ventilation and nebulization).

2 SVFN - ambiente sem ventilação e nebulização (environment with no ventilation and nebulization).

3 EXT - ambiente externo (outside environment). 
Tabela 4 - Valores médios de freqüência respiratória e temperatura retal para os respectivos tratamentos (VFN e SVFN) e períodos (manhã e tarde)

Table 4 - Average values for breathing frequency and rectal temperature for the respective treatments (VFN and SVFN) and periods (morning and afternoon)

\begin{tabular}{|c|c|c|c|}
\hline \multirow[t]{2}{*}{$\begin{array}{l}\text { Período } \\
\text { Period }\end{array}$} & \multicolumn{2}{|c|}{$\begin{array}{c}\text { Tratamentos } \\
\text { Treatments } \\
\end{array}$} & \multirow[t]{2}{*}{$\begin{array}{l}\text { Média (Período) } \\
\text { Average (Period) }\end{array}$} \\
\hline & VFN & SVFN & \\
\hline \multicolumn{4}{|c|}{ Freqüência respiratória $(\mathrm{mov} / \mathrm{min})^{1}$} \\
\hline \multicolumn{4}{|c|}{ Breathing frequency } \\
\hline Manhã (Morning) & 26,62 & 33,53 & 30,08 \\
\hline Tarde (Afternoon) & 26,17 & 44,35 & 35,26 \\
\hline Média/dia (Day average) & $26,40^{\mathrm{B}}$ & $38,94^{\mathrm{A}}$ & \\
\hline \multicolumn{4}{|l|}{ Temperatura retal $\left({ }^{\circ} \mathrm{C}\right)^{2}$} \\
\hline \multicolumn{4}{|l|}{ Rectal temperature } \\
\hline Manhã (Morning) & 38,06 & 38,23 & $38,16^{\mathrm{B}}$ \\
\hline Tarde (Afternoon) & 38,43 & 38,70 & $38,57^{\mathrm{A}}$ \\
\hline Média/dia (Day average) & 38,26 & 38,47 & \\
\hline
\end{tabular}

${ }^{1}$ Médias, na linha, seguidas de letras diferentes, diferem $(P<0,05)$ pelo teste Tukey (Means in the same line followed by different letters are different by Tukey test).

2 Médias, na coluna, seguidas de letras diferentes, diferem entre si $(P<0,05)$ pelo teste Tukey (Means in the same column followed by different letters are different by Tukey test).

a 20 respirações por minuto. Esse autor sugere que a primeira resposta dos suínos, quando expostos à temperatura acima do limite superior da zona de conforto térmico, é o aumento da taxa respiratória.

Os tratamentos não influenciaram $(\mathrm{P}>0,05)$ a TR das fêmeas gestantes. No entanto, observou-se efeito $(\mathrm{P}<0,05)$ dos períodos (manhã e tarde) sobre a TR, devendo-se ressaltar que, no período da manhã, a TR foi menor $(\mathrm{P}<0,05)$ que no período da tarde. No entanto, o maior valor de TR observado no período da tarde ainda se encontra dentro da normalidade para a espécie suína, que, segundo Hannas (1999), está em torno de $38,6^{\circ} \mathrm{C}$.

Os valores médios dos porcentuais de parto, retorno ao estro, abortamento, tamanho da leitegada, natimortos e mumificados, correspondentes aos animais mantidos nos dois tratamentos (VFN e SVFN), encontram-se na Tabela 5. Não foi observada diferença $(P>0,05)$ nos parâmetros reprodutivos avaliados para os animais de ambos os tratamentos estudados.

Os resultados obtidos permitem inferir que, embora o sistema de ventilação forçada associada à nebulização tenha promovido melhor condição ambiental dentro do galpão, esta não foi suficiente para alterar $(\mathrm{P}>0,05) \mathrm{a}$ eficiência reprodutiva das porcas.

Esses resultados estão de acordo com aqueles obtidos por Paterson et al. (1978), Stork (1979) e Love et al. (1995), que avaliaram o efeito da temperatura no início da gestação sobre a eficiência reprodutiva de fêmeas suínas. Stork (1979) observou fertilidade de $75 \%$ e taxa de retorno ao estro de $15 \%$, quando os animais foram expostos a temperaturas acima de $23^{\circ} \mathrm{C}$, por mais de três dias no período inicial da gestação. Love et al. (1995) encontraram taxas de parto de $71,3 \%$, quando as porcas foram cobertas em um período do ano no qual a temperatura média estava em torno de $28,5^{\circ} \mathrm{C}$. Patterson et al. (1978) observaram taxa de retorno ao estro de $14,4 \%$, quando as porcas foram submetidas à temperatura de $28 \mathrm{a}$ $31^{\circ} \mathrm{C}$, durante a primeira semana de gestação. Adicionalmente, quando os autores avaliaram a eficiência reprodutiva de porcas cobertas, em um período do ano no qual a temperatura não excedeu a $20^{\circ} \mathrm{C}$, obtiveram melhores resultados reprodutivos.

No presente trabalho, apesar da diferença na temperatura do ar nos horários mais quentes do dia (10 e 14 horas) entre os tratamentos VFN e SVFN (Tabela 3), constatou-se que, em ambos os ambientes, as temperaturas excederam $23^{\circ} \mathrm{C}$ e, em alguns dias, atingiram valores acima de $28^{\circ} \mathrm{C}$, sendo similares, portanto, às condições de temperaturas encontradas nos trabalhos relatados anteriormente. Entretanto, neste estudo, foi avaliada a TR dos animais que se encontravam em normotermia (temperatura corporal $<39,5^{\circ} \mathrm{C}$ ), não estando, provavelmente, submetidos a uma condição muito severa de estresse por calor.

Os resultados obtidos quanto às taxas de parto e retorno ao estro foram inferiores aos apresentados por Brandt et al. (1995) e Bortolozzo et al. (1997). Estes pesquisadores observaram que porcas nas 
Tabela 5 - Valores médios de porcentagem de parto, de retorno ao estro e de abortamentos, e valores médios de leitões nascidos, leitões nascidos vivos, natimortos e mumificados por porca, para os respectivos tratamentos (VFN e SVFN)

Table 5 - Avearage values for parturition, return to oestrus and abortion percentage and for total of born piglets, alive born, stillborn and mummified per sow, for the respective treatments

\begin{tabular}{|c|c|c|}
\hline \multirow[t]{2}{*}{$\begin{array}{l}\text { Parâmetros } \\
\text { Parameters }\end{array}$} & \multicolumn{2}{|c|}{$\begin{array}{c}\text { Tratamento } \\
\text { Treatment }\end{array}$} \\
\hline & VFN & SVFN \\
\hline Número de coberturas & 46 & 49 \\
\hline Number of matings & & \\
\hline Número de partos & 36 & 37 \\
\hline $\begin{array}{l}\text { Number of parturition } \\
\text { Taxa de parto }(\%)\end{array}$ & 78,3 & 75,5 \\
\hline $\begin{array}{l}\text { Parturition (\%) } \\
\text { Número de retorno ao estro }\end{array}$ & 7 & 8 \\
\hline $\begin{array}{l}\text { Number of return to oestrus } \\
\text { Retornos ao estro (\%) } \\
\text { Return to oestrus (\%) }\end{array}$ & 15,2 & 16,3 \\
\hline Retorno ao estro em intervalos regulares (\%) & 6,5 & 8,2 \\
\hline Retorno ao estro em intervalos irregulares (\%) & 8,7 & 8,2 \\
\hline $\begin{array}{l}\text { Return to oestrus on irregular interval (\%) } \\
\text { Número de abortamentos } \\
\text { Number of abortion }\end{array}$ & 2 & 3 \\
\hline $\begin{array}{l}\text { Taxa de aborto }(\%) \\
\text { Abortion }(\%)\end{array}$ & 4,3 & 6,1 \\
\hline $\begin{array}{l}\text { Total de leitões nascidos } \\
\text { Total of born piglets }\end{array}$ & 9,92 & 10,41 \\
\hline $\begin{array}{l}\text { Leitões nascidos vivos } \\
\text { Alive born piglets }\end{array}$ & 9,28 & 9,57 \\
\hline $\begin{array}{l}\text { Natimortos } \\
\text { Stillborn }\end{array}$ & 0,44 & 0,54 \\
\hline $\begin{array}{l}\text { Mumificados } \\
\text { Mummified }\end{array}$ & 0,19 & 0,30 \\
\hline
\end{tabular}

mesmas condições de normotermia encontradas neste trabalho $\left(\mathrm{TR}<39,5^{\circ} \mathrm{C}\right)$ apresentaram melhores resultados reprodutivos. Bortolozzo et al. (1997) observaram taxas de parto e de retorno ao estro de 82,8 e $9,2 \%$, respectivamente, e Brandt et al. (1995), taxa de retorno ao estro de 7,4\%. Esses autores encontraram resultados de taxa de parto e de retorno ao estro semelhantes aos obtidos neste trabalho, quando as porcas apresentaram hipertermia $\left(39,5^{\circ} \mathrm{C}\right)$. Assim, além da temperatura ambiente, outros fatores associados podem levar a falhas reprodutivas.

Os resultados de taxa de abortamento obtidos foram semelhantes aos observados por Domínguez et al. (1996), os quais encontraram altas taxas de abortamento para porcas cobertas no período de outono. Segundo Almond (2000), as causas do problema não estão definidas, mas acredita-se que uma combinação de fatores ambientais, nutricionais e de manejo pode predispor ao aparecimento dos abortamentos.

Quanto ao tamanho da leitegada, número de leitões nascidos vivos, natimortos e mumificados, também não foi observada diferença $(\mathrm{P}>0,05)$ entre os tratamentos, o que, segundo Sesti \& Sobestiansky (1998), está dentro do recomendado para produções suinícolas. Os resultados apresentados também estão de acordo com os de Domínguez et al. (1996), que observaram resultados semelhantes de tamanho da leitegada $(9,6)$, número de leitões nascidos vivos $(9,2)$ e natimortos $(0,6)$ para porcas cobertas no outono, no inverno e na primavera. Somente encontraram piores resultados para tamanho da leitegada $(8,4)$, leitões nascidos vivos $(8,2)$ e natimortos $(0,87)$, quando as porcas foram cobertas no verão.

$\mathrm{Na}$ Tabela 6 estão os valores médios de progesterona, obtidos do plasma sangüíneo das porcas no $6 \underline{0}$ e no $13 \underline{0}$ dia pós-cobertura, para os respec- 
Tabela 6 - Valores médios de progesterona $(\mathrm{ng} / \mathrm{mL})$ no 6 ㅇ e 13으 dia de gestação, para os respectivos tratamentos (VFN e SVFN)

Table 6 - Average values of progesterone $(\mathrm{ng} / \mathrm{mL})$ on $6^{\text {th }}$ and $13^{\text {th }}$ day of gestation, for the respective treatments (VFN and SVFN)

\begin{tabular}{|c|c|c|c|}
\hline \multirow[t]{2}{*}{$\begin{array}{l}\text { Dias de gestação } \\
\text { Days of gestation }\end{array}$} & \multicolumn{2}{|c|}{$\begin{array}{l}\text { Tratamentos } \\
\text { Treatments }\end{array}$} & \multirow[t]{2}{*}{$\begin{array}{l}\text { Média } \\
\text { Average }\end{array}$} \\
\hline & VFN & SFVN & \\
\hline $\begin{array}{l}\text { Sexto } \\
\text { Sixth }\end{array}$ & 14,40 & 13,49 & 13,95 \\
\hline $\begin{array}{l}\text { Décimo terceiro } \\
\text { Thirteenth }\end{array}$ & 26,75 & 23,66 & 25,21 \\
\hline $\begin{array}{l}\text { Thirteenth } \\
\text { Média } \\
\text { Average }\end{array}$ & 20,58 & 18,58 & \\
\hline
\end{tabular}

tivos tratamentos (VFN e SVFN). Não foi observado efeito dos tratamentos $(\mathrm{P}>0,05)$ sobre os níveis de progesterona no $6 \underline{0}$ e 13 o dia de gestação. Os níveis séricos obtidos de progesterona estão de acordo com os encontrados por Hoagland \& Wettemann (1985) e Varley e Prime (1993). Hoagland \& Wettemann(1984), trabalhando com porcas em estresse por calor e em conforto térmico, encontraram valores médios de progesterona plasmática no 13 o dia de gestação em torno de $26 \mathrm{ng} / \mathrm{mL}$. Varley \& Prime (1993), utilizando o mesmo manejo alimentar pós-cobertura deste trabalho, encontraram, no sexto dia de gestação, valores médios de progesterona em torno de $13 \mathrm{ng} / \mathrm{mL}$, porém eles não avaliaram o ambiente térmico. De acordo com Anderson (1988), os valores de progesterona encontrados estão dentro dos níveis normais para fêmeas suínas em gestação.

\section{Conclusões}

Para a época do outono, na região de Ponte NovaMG, a utilização do sistema de acondicionamento térmico constituído por ventilação e nebulização não incrementa a eficiência reprodutiva.

\section{Literatura Citada}

AGROCERES. Condição corporal das fêmeas reprodutoras. Acessado em: 10/02/2000. (http://www.agroceres.com.br). 2000.

ALMOND, G.W. Seasonal infertility in female pigs. Acessado em: 09/08/2000 (http://mark.asci.ncsu.edu/reproduction/ summer/seasonal.htm). 2000.

ANDERSON, G.W. Suínos. In: HAFEZ, E.S.E. (Ed.) Reprodução animal. São Paulo: Manole, 4.ed. 1998. p.412-446.
BAÊTA, F.C.; SOUZA, C.F. Ambiência em edificações rurais - conforto animal. Viçosa, MG: Universidade Federal de Viçosa, 1997. 246p.

BORTOLOZZO, F.P.; WENTZ, I.; BRANT, G. et al. Influência da temperatura corporal sobre a eficiência reprodutiva em fêmeas suínas. In: CONGRESSO BRASILEIRO DE VETERINÁRIOS ESPECIALISTAS EM SUÍNOS, 1997, Foz do Iguaçu. Anais... Foz do Iguaçu: ABRAVES, 1997. p.281-282.

BRANDT, G.; WENTZ, I.; BORTOLOZZO, F.P. et al. Influência da temperatura corporal sobre a eficiência reprodutiva da fêmea suína. In: CONGRESSO BRASILEIRO DE VETERINÁRIOS ESPECIALISTAS EM SUÍNOS, 1995, Blumenau. Anais... Blumenau: ABRAVES, 1995. p.129.

BUFFINGTON, D.E.; COLAZZO-AROCHO, A.; CANTON, C.H. et al. Black globe-humidity index (BGHI) as comfort equation for dairy cows. Transaction of the ASAE, v.24, p.711-14, 1981.

DOMÍNGUEZ, J.C.; PEÑA, F.J.; ANEL, L. et al. Swine summer infertility syndrome in north west spain. Veterinary Record, v.27, p.39-94, 1996.

FRIEND, D.W.; BROWN, R.G. Blood sampling form suckling piglets. Canadian Journal of Animal Science, v.51, p.547$549,1971$.

HANNAS, M.I. Aspectos fisiológicos e a produção de suínos em clima quente. In: SILVA, I.J.O. (Ed.) Ambiência e qualidade na produção industrial de suínos. Piracicaba: Fundação de Estudos Agrários Luiz de Queiroz, 1999. p.1-33.

HOAGLAND, T.A.; WETTEMANN, R.P. Influence of elevated ambient temperature after breeding on plasma corticoids, estradiol and progesterone in gilts. Theriogenology, v.22, p.15-24, 1984.

HURTGEN, G.P.; LEMAN, A.D. Seasonal influence on fertility of sows and gilts. Journal Animal Veterinary Medical Association, v.177, p.631-635, 1980.

LEE, D.H.K.; PHILLIPS, R.W. Assesment of the adaptability of livestock to climatic stress. Journal of Animal Science, v.7, n.4, p.391-425, 1948.

LOVE, R.J. Definition of a seasonal infertility problem in pigs. Veterinary Record, v.103, p.443-446, 1978.

LOVE, R.J. Seasonal infertility in pigs. Veterinary Record, v.109, p.407-409, 1981.

LOVE, R.J.; KLUPIEC, C.; THORNTON, E.J. et al. An interaction between feeding rate and season affects fertility of sows. Animal Reproduction Science, v.39, p.275-284, 1995.

MOURA, D.J. Ventilação na suinocultura. In: SILVA, I.J.O (Ed.) Ambiência e qualidade na produção industrial de suínos. Piracicaba: Fundação de Estudos Agrários Luiz de Queiroz, 1999. p.149-179.

NÄÄS, I.A. Influência do ambiente na resposta reprodutiva de fêmeas. In: SIMPÓSIO INTERNACIONAL DE REPRODUÇÃO E INSEMINAÇÃO ARTIFICIAL EM SUÍNOS, 7., 2000, Foz do Iguaçu. Anais... Foz do Iguaçu: Embrapa Suínos e Aves, 2000. p.253-262.

PATTERSON, A.M.; BAKER, I.; LINDASAY, D.R. Summer infertility in pigs: its incidence and characteristics in an australian comercial piggery. Australian Journal Experimentaly Husbary, v.18, p.698-701, 1978.

ROZEBOOM, K.; SEE, T.; FLOWERS, B. Coping with seasonal infertility in the herd: part $I, 2000$. Acessado em: 09/08/2000 (http://mark.asci.ncsu.edu/Swine_News/ 2000/sn v2303.htm).

SESTI, L.A.C.; SOBESTIANSKY, J. Aspectos da produtividade.

R. Bras. Zootec., v.32, n.4, p.854-863, 2003 
In: SUINOCULTURA INTENSIVA. PRODUÇÃO, MANEJO E SAÚdE DO REBANHO, 1998, Brasília. SPI... Concórdia: Embrapa Suínos e Aves, 1998. p.27-43.

STORK, M.G. Seasonal reproductive inefficiency in large pig breeding units in Britain. Veterinary Record, v.104, p.49-52, 1979.

TURCO, S.H.N. Modificações das condições ambientais de verão em maternidades de suínos. Viçosa, MG: Universidade Federal de Viçosa, 1993. 59p. Dissertação (Mestrado em Zootecnia) - Universidade Federal de Viçosa, 1993.

UNIVERSIDADE FEDERAL DE VIÇOSA-UFV.SAEG. Sistema para análises estatísticas e genéticas. Versão 8.0. Viçosa, MG: 1999. 159p. (Manual do usuário).

Van der LENDE, T. Mortalidade embrionária e fetal em suínos: causas, conseqüências e como prevenir estas perdas. In: SIMPÓSIO INTERNACIONAL DE REPRODUÇÃO E INSEMINAÇÃO ARTIFICIAL EM SUÍNOS, 7., 2000, Foz do Iguaçu. Anais... Foz do Iguaçu: Embrapa Suínos e Aves, 2000. p.243-252.
VARLEY, M.A.; PRIME, G.R. The effect of food intake on prolificacy and plasma progesterone concentrations in multiparous sows. Livestock Production Science, v.34, p.267-279, 1993

VIANELLO, R.L.; ALVES, R. Meteorologia básica e aplicações. Viçosa, MG: Universidade Federal de Viçosa, 1991. 449p.

WETTEMAN, R.P.; BAZER, F.W. Influence of environmental temperature on prolificacy of pig. Journal of Reproduction and Fertility, v.33, p.199-208, 1985.

Recebido em: 27/02/02

Aceito em: 20/11/02 